\title{
Ethical Responsibilities Towards Dogs: An Inquiry into the Dog-Human Relationship
}

\author{
Kristien Hens
}

Accepted: 11 August 2008/Published online: 22 August 2008

(C) Springer Science+Business Media B.V. 2008

\begin{abstract}
The conditions of life of many companion animals and the rate at which they are surrendered to shelters raise many ethical issues. What duties do we have towards the dogs that live in our society? To suggest answers to these questions, I first give four possible ways of looking at the relationship between man and dog: master-slave, employer-worker, parent-child, and friend-friend. I argue that the morally acceptable relationships are of a different kind but bears family resemblances to the latter three. As dogs are beings with an interest in their wellbeing, society has certain duties towards the dogs, which can be translated in legislation. But human beings also have special responsibilities towards the dogs they take into their care. Such responsibilities entail caring for their emotional and physical welfare, but also ensuring a bond of trust, which should not easily be broken.
\end{abstract}

Keywords Dog - Companion animals - Environmental ethics · Ethics of care

\section{Introduction}

Although research has shown that a substantial amount of money is spent on food and accessories for dogs (American Veterinary Medical Association (AVMA) 2002), this does not correlate with the welfare of these animals in present-day society. For example, in 2004, 38,586 dogs were accepted in Belgian animal shelters, an increase by 2,000 dogs compared to 2003. The excesses of present-day dog breeding have been documented by authors such as Stafford (2007) and Derr (2004), who have pointed out that a focus on the aesthetic "qualities" of a breed may lead to reproductive problems (bitches that have to give birth through caesarean), skin disorders, the proliferation of hip dysplasia, and other inheritable defects.

K. Hens $(\square)$

Faculty of Medicine, Centre of Biomedical Ethics and Law, Katholieke Universiteit Leuven,

Kapucijnenvoer 35, Bus 7001, 3000 Leuven, Belgium

e-mail: Kristien.hens@med.kuleuven.be 
In this article, I shall investigate which responsibilities we have towards individual dogs and dogs as a species. I shall also discuss whether we have a responsibility towards breeds of dogs. I shall argue that our responsibilities not only arise from duties towards animals in general: given the special status of dogs in our families, we also have extra responsibilities towards our own dogs. Moreover, those that claim to love a breed have responsibilities towards that breed as well. I shall first describe theories about the history and the nature of man's relation to dogs, and then lay down the specific responsibilities we have towards dogs as individuals and as species. I shall finish by giving some general conclusions on how these responsibilities could be translated into practices.

\subsection{History and Nature of Our Relation to Dogs}

In order to tackle the issue of which responsibilities we have towards dogs, we shall investigate some of the current views on the dog-human relationship. Common views are that of the dog as slave, as replacement child or as friend, and as worker.

Some animal rights advocates thinks that companion animals are slaves, in need of liberation. For example, Ingrid Newkirk, president of PETA (People for the Ethical Treatment of Animals) states that in an ideal world, breeding of companion animals would no longer be allowed: “... as the surplus of cats and dogs (artificially engineered by centuries of forced breeding) declined, eventually companion animals would be phased out, and we would return to a more symbiotic relationship — enjoyment at a distance" (Hitt 1991). She expresses the view that companion animals are the result of human domination, and should be restored to their original wild status. Following this logic, an ethics of companion animals is redundant: an animal ethic should be consistent for each type of human-animal relation. In the terminology of some animal rights advocates, this would mean stopping breeding companion animals. In the end, only wild animals would remain. The lawyer and animal rights advocate Francione (2000) has a similar view. His theory is based on the assumption that one of the basic rights of human beings and animals alike is the right not to be treated as property. By its very nature, property cannot have real rights. However, since animals and humans alike have the same interest in not suffering, they should be treated according to the principle of equal consideration in this respect. Therefore, they should not be seen as property. Francione explicitly sees companion animals as property: "Indeed, it is precisely because pets are our property that we can choose to value them as more than economic commodities" (Francione 2000, p. 77). His solution approximates the statement of Newkirk: They both state that we are allowed to adopt abandoned pets from shelters, but we should not bring more of them into existence through breeding. They both assume the human-companion animal relationship is unilateral, and that it originates from an act of will of human beings, who once decided to take animals from their natural environment and make them into slaves. A similar concept is the theme of Tuan's seminal work Dominance and Affection: The Making of Pets (1984). Tuan uses the word "pet" in its broadest sense: for him it also refers to plants, gardens, water, animals, and even children, women, dwarves, and servants. These relationships are all characterized by a desire to dominate, which in itself stems from man's inherent insecurity towards the unruly forces of nature. It is not necessarily an aggressive type of domination, but one closely linked with affection and playfulness. In Tuan's vision, pets receive lavish care and much money is spent on them, but they exist only for human pleasure and convenience. The example par excellence of exercising human dominion is dog training, in which the power to dominate and humble another living being is demonstrated openly. 
Hence for Tuan, the domestication of pets was at least a semi-conscious process: mankind deliberately chose wild animals to breed pets out of its psychological need to dominate. He also situates the practice entirely in the realm of the cultural rather than the natural. Companion animals are the perfect example of the excesses of culture, and the call for total liberation seems justified. Some, however, consider domestication not to be such a oneway affair. Natural evolution may not have stopped and culture started with the emergence of the first dog. And does the term slave accurately cover the relationship many people have with their companion animals?

Dogs are descendent from wolves but the molecular distance between wolf and dog is so great that some believe they must have separated around 100,000 BP (Vilà et al. 1997). The best known story about the origins of dog keeping comes from Konrad Lorenz in his book Man Meets Dog (Lorenz 1988). He describes how a little cave-dweller girl finds a wolf puppy and brings it home. The puppy grows older and proves to be a valuable asset as a hunting partner. Clutton-Brock (1999) describes a similar process. She states that the process of taming started around 15,000 years ago, when dogs and wolves became also morphologically different, but specifies that it is difficult to assess how much has changed in the relationship between dogs and humans. She thinks the basis of the success of the relationship is the fact that both humans and dogs are social animals, and that some reciprocal affection is possible. Also, the fact that hunting techniques of both species complement each other has lead to the success of the domestication. A slightly different view of domestication of the dogs is formulated by Raymond Coppinger (Coppinger and Coppinger 2001, see also Koler-Matznick 2002). He investigated village dogs around the world, and found some common characteristics: they are smallish but uniform in size and appearance, and do not really have "owners," He sees them as modern examples of the canid "missing link" and describes how wolves probably already became dogs, and adapted themselves to the village niche, before they became truly domesticated. This line of reasoning is continued by Budiansky (1997), who claims that all domestication was initiated by the animals and that it is a natural rather than a cultural phenomenon. According to him, animals are not dependent on human beings because we chose to confine them, but by their evolutionary history. There is a "contract" between domesticated animals and humans. In return of shelter and an easy life, human beings can put them to use in whatever ways seem appropriate.

The discussion on the domestication of animals is not settled. Some claims of the adherents of the contract theory, such as Budiansky, are exaggerated. Few would accept the naturalness of battery chicken. With the dog, however, I think there are different issues at stake. If we want to think about a proper ethics towards dogs, we must do so in the context of the dog and its specific niche, which is the human world, not using some vision of the dog as a wild animal. I do not agree with Rolston III when he states that "A cow does not have the integrity of a deer, or a poodle that of a wolf" (Rolston III 1993). He believes that the adoption of pet animals by mankind works to the detriment of these animals, as they lose some of their wildness and therefore their integrity. But since the natural environment of dogs is, and always has been, human culture, this seems not to apply. If we consider dogs as a species of its own right, there is no wildness to lose: if the integrity of an animal is defined by the niche in which it lives, dogs, as members of human cultures, are as integer as a wolf in the wild. The notion of naturecultureness that Donna Haraway borrowed from Bruno Latour may be useful here: In her books The Companion Species Manifesto: Dogs, People, and Significant Otherness (Haraway 2003) and When Species Meet (Haraway 2008), she investigates the relationship dog-owner. The first book is intended as a followup of her work “A Cyborg Manifesto" (Haraway 1991). In this work, she wanted to break 
away from traditional Marxist thinking, which saw a progressing transformation of nature to culture by mankind. She uses the imagery of the cyborg, which is a hybrid of machine and organism, as a trope for the fact that present-day reality is not about strict distinctions. The ancient dividing lines between nature and culture, human and animal, organic and nonorganic, physical and non-physical are blurred: This creates new possibilities that we should embrace rather than flee from. Human beings are cyborg-like beings, who cannot return to nature nor escape it totally. In her more recent works, she thinks that perhaps dogs are a better metaphor for this kind of existence.

Cyborgs and companion species each bring together the human and non-human, the organic and technological, carbon and silicon, freedom and structure, history and myth, the rich and the poor, the state and the subject, diversity and depletion, modernity and postmodernity, and nature and culture in unexpected ways. Besides, neither a cyborg nor a companion animal pleases the pure of heart who long for better protected species boundaries and sterilization of category deviants. (Haraway 2003, p. 4)

Dogs are examples of entities that can no longer be reduced to their natural essence, nor can they be seen as purely cultural constructs. Dogs, as stressed by Derr (2004) as well, are animals that do not exist outside human cultural context.

Moreover, man's relationship to dogs is not one of dominion but of a two-way interaction. The success of the human-dog relationship suggests that it is more than just one of master versus slave. Despret (2004) describes the process of domestication as anthropo-zoo-genesis: between human and non-human animal, domestication becomes possible in the context of trust. Each player fulfills certain expectations, but to fulfill these expectations is not the same as to obey these expectations, as would do a mere slave. She describes how both man and animal are active partners in the process of domestication, and are transformed by the availability of the other. This may not be applicable to all animals that are used as "pets" today (think of pet fish, snakes or spiders kept in aquaria or terrariums), but it makes sense to view the domestication of dogs in this way. Awareness of this special position of dog as a companion species can shed valuable light on the way they should be treated. True, there are many examples of humans who treat their dogs as mere tools or slaves. In some branches of dog sports, there are owners who would do away with a dog when he or she does not or does no longer perform. But repressive training techniques destroy the dog-human relationship rather than build it. The current trend in dog training is to focus on bond-making rather than on mechanistic tools of rewards and punishment. As Suzanne Clothier states in Bones would rain from the sky: "Each relationship with an animal and a human is a bridge uniquely shaped to carry only those two, and so must be crafted by them" (Clothier 2002, p. 29). For Clothier, it is the quality of the relation between dog and human that guarantees results, not only applying the right learning theories. It may be tempting to view her training techniques as very modern, but in her article on hunting dogs, Sophia Menache quotes medieval authors who already stress proper communication with the dogs to be trained: "You should speak kindly [with your dog], taking care of avoiding any anger against him, but to be as gentle as possible, to lay your hand on his head, and to kindly caress him" (Menache 2001, p. 53). It is indeed questionable whether the relationship dog-human would have been so successful if they were merely man's tools.

If dogs are not man's tools or slaves, should we consider the relationship as one of employer-employee? There are indeed some resemblances to this relationship. This is most obvious in the relationship of working dogs with their owner. The guide dog and its blind owner, the police officer and his or her K-9 security dog, the hunter and his or her hunting 
dog, these seem examples of how dogs are employees that work for their daily bread. Even the dog that is kept as the sole companion of an elderly person, could be seen as a modern version of a "lady-companion." In television programs and news stories, the working dog is often presented as the example of the success of the human-animal relationship. And indeed, for many dogs, the fact that they have certain tasks to perform gives them great satisfaction. Like with human subjects, it seems that a job suitable to their capacities, which is well rewarded, gives dogs a sense of wellbeing and "happiness" that is absent in dogs that are idle most of the time. On the other hand, there are substantial differences with this type of relationship as well. The most striking one is the absence of a contract between handler and working dog. The dog is just assumed to perform its duty, and has no say in any compensation or in the type of work they have to perform. This can result in certain malpractices. Some have argued (Serpell et al. 2003) that assistance and therapy dogs are often forced to perform under suboptimal circumstances: before their training and placement in a home, they are raised in sterile kennels. Often inhumane training methods, that use lavish punishment, are used in training assistance dogs for the blind. Also they have to switch owners a couple of times before they are finally placed with their "end-users," which can be detrimental to a dog's mental health. K-9 police dogs are in many cases not taken home to families after the work is done, but are put in kennels and given no playtime or time off duty. In these cases, the relationship becomes more one of master-slave than of employer-employee. But these aberrations should not be considered the rule. Overall, although the relationship is not strictly speaking that of an employer and his or her employee, there are family resemblances, as dogs are made to do many different tasks for their humans. Moreover, if dogs were not willingly working for human beings in the first place, the relationship would not have existed in its current form. At least, the resemblances with the employer-employee relationship can shed a light on our moral obligations towards dogs.

Should we describe the relationship between dog and human as one of human-child? Often, dogs are considered as replacement children, a perversion of a relationship that should have been directed to another human. Such a view is understandable if we look at the current practice of dressing dogs in fancy clothes, designer jewelry especially made for dogs, nail varnish for dogs. But Anthony L. Podberscek and Samuel D. Gosling state that there is no proof that there is any significant difference in personality between pet-owners and non-pet owners, although there may be differences between owners of different types of pets. Equally it is not true that pets are usually kept by solitary people as substitutes for normal human contact (Podberscek et al. 2001). Furthermore, most owners of pets are part of a family, often with children. It seems that there is something extra people want from a companion animal, a need for non-human contact that is complimentary to human relations (Endenburg 2001). There are also many differences when comparing the parent-child relationship and the pet-owner relationship. Most people would go to extremes to guarantee the well-being of their children, financially, emotionally and even morally: parental love defies many boundaries that exist in society. Also, the idea of outliving one's own children is close to the unthinkable. Dogs, on the other hand, have life spans that are far shorter than the natural lifespan of a human being. They are to some extent interchangeable. Although the death of a beloved pet can be a very emotional and traumatizing experience, it still falls in the realms of what is to be expected. Also, parents make certain decisions for their children in the light of the autonomous beings they will once become. Parenting is finding equilibrium between restricting a child's freedom out of concern of their wellbeing, and allowing them more and more autonomy as they grow older. Dogs, although some more restrictions are put on puppies than on fully trained dogs, will never 
grow into completely autonomous beings that are allowed to make their own choices independent of their owners. Hence, the relationship will always be somewhat paternalistic. As Burgess-Jackson argues "A responsible human companion restrains a dog's impulses-limits its liberty, frustrates its will- order to protect it from known or foreseeable dangers" (Burgess-Jackson 1998, p. 183). However, there are also some striking similarities: both children and companion animals come into existence because their parents or owners do so wish. They both are welcomed in human families and are to some extent at the mercy of these families. Strikingly, domesticated dogs expose much more juvenile traits than their wild ancestors: they have softer appearances; they play and enjoy being touched and cuddled. As stated by Burgess-Jackson (1998) they seem more vulnerable than wild animals. The fact that we have welcomed them voluntarily in our household, together with this vulnerability, implies that we have responsibilities towards them that extend the responsibilities we have towards wild animals and are more like the responsibilities we have towards our children.

Are dogs our friends? With a relationship of friendship, there are again major differences: dogs are only for a short period of time in our lives, they are to some extent replaceable and they are at our mercy with regard to medical care and euthanasia. Also, some might argue that having a language in common is a prerequisite of true friendship. However, just as in a bond of friendship, companion animals and their owners may find great comfort in each other's company. Many people claim to love their animals and believe that they are loved back by them. Dog owners can have a true bond with their animals, even if this bond is of a somewhat different nature than that between human beings. Derr (2004) defines the friendship between dogs and humans as follows:

At its core, the relationship between human and dog is an uncluttered one involving two distinct animals who just happen to understand each other at a basic level. That

more than a few people end up preferring dogs to humans can come to no surprise.

(Derr 2004, p. 339)

I have argued that the relationship between man and his or her dog is a reciprocal one: a mere act of dominion can not explain the success and the history of the relation. Moreover, there are family resemblances to both the human-child relationship as well as to the bond of friendship and as to the relationship employer-employee provide some guidance as to which responsibilities we have towards our dogs.

\section{Our Moral Obligations to Dogs}

In the first part of this article, I have argued that dogs occupy a special position. They are non-human animals with a natural history. Hence we should not treat them less than we would other animals. But dogs are typical naturecultural animals, living in relationship with human beings. Because of this relationship, we have extra responsibilities to the dogs we take up in our families. Moreover, as Haraway states (Haraway 2008), a relationship with a dog is also a relationship to a kind of dog, a breed. Do we have extra duties towards breeds? Which responsibilities do we have towards dogs as individual animals, as part of the species canis lupus familiaris, and as part of a breed?

First, what intrinsic responsibilities do we, as a society and as individuals within that society, have towards animals as individuals and species? In many theories, either individuals or species or the ecosystems as a whole are considered. A useful framework is that of Lawrence Johnson (Johnson 1991). He reconciles these views using the concept of 
interests. He believes that the moral universe must extend beyond the human sphere, and bases his environmental ethics on the fact that entities have moral significance because they have interests. The interests that are morally significant are not necessarily hedonistic: also entities that have no experiences and mental state have them. But all entities that have an interest in their own wellbeing have moral status. This applies to individuals, as they must be respected in what is appropriate for their wellbeing. As he states "the wolf must be respected as a wolf." Along the same lines, we could say that the dog must be respected as a dog. We shall discuss later on what this means. But also, according to Johnson, species have morally significant interests. For him, entities with interests have moral significance. As a species is also a living system, a life process that tries to achieve or maintain optimal states, it has interests. And these interests are not simply an aggregate of the individuals belonging to that species. Since a species is such an ongoing, organic whole, it is a moral agent in itself. It has interests in its own survival, but also in surviving in circumstances adequate to its needs. Hence, a species that only survives in zoos is morally wronged, even if the individuals are well taken care of. For Johnson, this requires that we have the responsibility to provide the opportunity to fulfill the need of a species' nature in an appropriate environment, and "to maintain itself as a species in a coherent, integrated, functional, ongoing whole with a particular interest" (Johnson 1991, p. 180). As both individuals and species are moral agents in their own right, there is no straightforward way to assign priorities: in some cases the interest of one individual will outweigh the interest of another individual. Also, the interest of a species may outweigh that of another individual, even if that individual is a human being. I shall use the idea of interest in wellbeing when I discuss responsibilities towards dogs as individuals and species.

But dogs, as discussed above, are also animals that live in families and are part of our closest communities. We can use the intrinsic moral status of dogs and their species to define general duties a society and individuals within that society may have to them. But the very act of taking them into our families and have a relationship with specific dogs generates specific responsibilities toward those dogs. That companion animals are part of communities and that this generates special responsibilities apart from the responsibilities we have towards animals in general is no new thought. In his article on companion animals, Clark (1995) describes how in the beginnings of mankind, personal attachments to relatives and friends were the basis of moral understanding, and it was only after the expansion of different empires that there was a need of more extended ethics. But personal attachments still matter. Since there is no essential boundary between humans and nonhumans, it is unclear why animals that we are close to should not be included in this ethics of personal attachment. Also Callicott (1998), Midgley (1984) and Gruen (1993) apply a communitarian approach to animal ethics. Midgley describes how man does not naturally exist in species isolation and how throughout the history of mankind human beings have been involved with non-human animals. She believes domestication was only so successful because individual bonds could be formed, something that I have also discussed in the first part of this article. And as Callicott states, membership of communities generates duties and obligations to other members, next to impersonal obligations we have towards individuals in general. To take into account what is most important, we must weigh two things: responsibilities towards members of more intimate communities are more important than impersonal obligations, but interests matter as well: stronger interests should take precedence over weaker interests. The intimacy of the relation and the severity of the interest should be weighted against one another. Burgess-Jackson (1998) applies a similar idea to companion animals. He does consider there to be a difference between responsibilities to "wild" or "stranger" animals and to the ones we have brought into our homes deliberately. 
He states that the act of forming a bond or relationship with a sentient being generates responsibilities to care for its needs. There is a bond of mutual trust between dog and human that should not be breached.

I believe that the mere fact that we accept one or more dogs as part of our family, generates specific responsibilities. I shall elaborate on what these responsibilities could be in the last part of this article.

A difficult question is the one about breeds of dogs. Should breeds of dogs exist or is only the quintessential mutt dog the standard? Current aberrations in breeding practices suggest the latter: what good could there be in a dog that can only deliver through cesarean or that has skin problems because of genetic defects? Some suggest that all breeds are the result of extensive selective breeding, starting at the end of the nineteenth century. Derr (2004), for example, states that until the rise of dog shows in the nineteenth century, what distinguished a type of dog was its way of working and its basic appearances. Breeds, as they exist now, seem to be an artificial creation of kennel clubs. Clutton-Brock (1995) describes how there are now over 400 breeds, but how several distinct breeds arose about 3,000-4,000 years ago. After that, in ancient Egypt one could, for example, find dogs of the greyhound type and dogs of the mastiff type, the latter used as hunting and guard dogs. In the Roman period, there were hunting dogs, guard dogs, sheep and lap dogs. In the middle ages there was a further differentiation of the different types of hunting dogs, for different game (deerhounds, wolfhounds, otterhounds). So the idea of dog breeds as such is not a modern invention and the result of modern manipulative breeding solely, although the emphasis on external aesthetic characteristics of the members of a breed may be. Haraway (2008) describes how she first thought purebred dogs as an aberration, but after being in close contact to bona fide breeders, she describes how you can fall in love with kinds as well as with individuals: the specific history and characteristics of a breed make it worth some dedication. I tend to agree with that: the loss of dog breeds with their specific capabilities does seem undesirable. Along the same lines, although survival of a breed of dog as such is probably subordinate to survival of dog as a species, one could argue that, in the lines of Johnson's theory of interests of species, that at least the healthier breeds have some interests in surviving in an adequate environment as well. How this could apply to ethical standards with regard to breeds I shall investigate in the next section.

\section{Application General and Special Responsibilities Towards Dogs}

My first question is whether we, as a society and as members of that society have special responsibilities towards dogs in general, regardless of any special relationships we might have with specific dogs or breeds. I shall take the ideas of Johnson that we described in the previous section: individual animals, and also species have an interest in their wellbeing. As such, individual dogs, and the dog as a species are moral agents whose wellbeing should be respected. As I have pointed out above, the wellbeing of a dog is not that of a wolf: a dog does not exist outside human culture. Although in many parts of the world dogs live a feral existence, they still do so in the proximity of human culture, and are predominantly scavengers (Coppinger and Coppinger 2001), hence not real "wildtypes." The link between dogs and human culture does not entail that a dog is less valuable than a wolf, but has value in itself as naturecultural being. The responsibilities we have as a society towards dogs can thus be situated in the fact that we should allow its members to take a proper place in society. Although it may seem that love of "pet animals" has risen significantly during the last century, this has also been accompanied with a diminishing of the life space 
and autonomy of dogs within our society. In the early twentieth century, it was common to see dogs wander freely. With the rising urbanization of the Western world, this has become less and less possible. Moreover, a growing number of people are less comfortable around dogs than the generations before them. This has led to laws requiring dogs to be on leash all the time, and the reduction of places where dogs can enter. Some dog therapists have argued that the reduction of dog-friendly places leads to behavioral problems in dogs, as they are not given the opportunity to socialize with other dogs and humans. It is society's responsibility towards dogs to accommodate for these changing situations by installing dog parks and off-leash walking routes. Also, fear or unease around dogs could be reduced, to the benefit of both parties, by special sessions in schools: children could be taught how to approach and handle dogs. Also laws should be extensive to reduce and control abuse of individual animals. Society should invest in proper shelters for abandoned dogs. Given the huge amount of dogs that end up in shelters, this also means offering information to the public as to what it means to own a dog, and to encourage dog adoption from shelters. As Spencer et al. (2006) argue, rules for companion animals should certainly be no less strict than those for farm animals. For example, the existence of puppy mills and the few regulations that exist in most countries to encourage responsible dog breeding suggest that in practice it is the other way around. A puppy mill is typically a dog breeding farm: the (as I will argue later) often misplaced desire of people to buy a cheaper puppy of a certain breed has lead to the existence of farms that house bitches in sheds and have them produce as many litters as possible. The lack of regulation in many countries concerning the amount of dogs a person can own or the number of litters a bitch can produce lead to excesses that have no respect for the dogs as beings with specific needs of companionship and playtime. Hence, seeing dogs as animals with an interest in their wellbeing also entails strict breeding regulation and the abandonment of mass dog breeding facilities altogether.

Do we have general responsibilities towards breeds of dogs? As we have discussed, the proliferation of breeds dates from the end of the nineteenth century, although there have always been types of dogs, adapted to specific purposes. I think duties of society towards breeds are less than towards individual dogs or the species "dog," but in general one could say that all types of dogs need to have space and opportunity to exercise their own characteristics. In this respect, dog sports such as agility, flyball, trailing, and tracking, which are great opportunities to provide for the working needs of certain breeds, could be encouraged.

There are also special responsibilities arising from bonds with specific dogs that exceed those towards dogs in general, be it individual or as a species. As we have pointed out, the relationship with a dog bears resemblance to the relationship we have to an employee, a friend, and a child, and certain responsibilities will overlap. I shall sum up some of the responsibilities I think we have towards dogs under our care, although the list is not exhaustive. For example, although we are not obliged to feed or vaccinate any "strange" dog not in immediate need for care, we should feed the ones that are in our family or that we employ as workers under our care with the food most appropriate to their health. They should be given sufficient medical care and should be vaccinated to prevent certain lethal diseases. Adopting a dog should be a conscious decision, preceded by a thorough reflection on the future welfare of the animal in question, as it means engaging in a relationship of trust. Taking in a dog to drop her at the local shelter right before the holiday season (or have her euthanized) violates the relation of trust, as pointed out by Johnson (1991). If a dog is adopted to function as a working dog, we should be aware that the dog may not fulfill that function according to the expectations. In such cases, it is the duty of the owner to still provide the dog with an adequate living. I believe it is only in exceptional cases 
morally allowed to dispose of a dog because it does not perform well. And even in that case it is the responsibility of the owner to make sure that the dog is rehomed in a suitable family.

Spencer et al. (2006) states that dogs are naturally pack animals: we are responsible that the dogs under our care, as companions or workers, have sufficient interaction with the rest of the family members and should not spend an entire day or night alone. Also, certain dogs have an innate desire to work and perform certain tasks: if we were to keep these dogs solely as companions around the house and playthings for the children, we cause them harm, however healthy and well fed the dog might be. The question of castration is a difficult one, as castration may seem to be an infringement of the animal's sexual integrity. However, Burgess-Jackson (1998) rightly states that if your dog reproduces, you must ensure that the needs of the resultant puppies are fulfilled. In many cases, this may be impossible. Moreover, the imperative to allow the animal to live a fulfilling life may be more compelling than the duty to preserve her sexual integrity. It may, for example, be more appropriate to castrate a dog than to separate her during several weeks a year from playmates.

As pointed out before, the relationship with a dog has family resemblance to the relation with a friend, a child, or an employee, but there are some striking differences too. The life span of a dog is typically much shorter than that of a human being, and the quality of life of a dog is very different from the quality of life of a human being. Hence, though I believe neither money nor time should be spared to give a dog a valuable life, there are limits. For example, no-one is obliged to sell his or her house or spend the money they saved for their children's education to put a dog on months long dialysis. I would assume the interests of the children here are more important than that of the dog. Moreover, a dog that has to spend the vast majority of the day on dialysis probably has a very limited quality of life. But it remains difficult to assess the latter for any other living being, be it human or non-human. Often we hear people sigh that a three-legged dog had better be euthanized. I believe it is dependent on the character of the dog whether this is actually true: a typical lap dog may be perfectly happy with one leg missing, but a working dog that is used to herding sheep all day may not be able to adapt to a more sedentary life style.

What personal responsibilities arise from the love of a certain breed of dog? I have argued before that I think that dog adoption from a shelter should be encouraged and preferred to buying a purebred puppy. I would like to nuance that here. As shown, types of dogs and love of these types have always existed, and breeds may have some interests in the continuation of their existence, similar (but more limited) than those of the species as a whole. Although the rise of the kennel club with its fixed breed standards may have contributed to the perception that breeds are in se unhealthy and should be done away with, I think people who make a conscious choice out of love for a specific breed should not be blamed either. However, the vast majority of dog owners just want a dog as a family pet. They may have at first sight a preference for a certain breed but this is often not founded on solid grounds ("we want a border collie because they are smart," "we want a labrador because they are so good with children"). These kinds of beliefs are misplaced. They are one of the main reasons for the existence of puppy mills and pet stores, which should be outlawed. I believe that people just looking for a nice family dog should be encouraged to look for a puppy or grown-up dog in dog shelters. The dog shelters themselves should be well equipped and have dog behaviorists employed to find the right dog for the right person. A wrong dog in a wrong family can be detrimental to the reputation of the dog species in general and to the trust in dogs of the humans involved. 
But, just as the species "dog" should not be allowed to become extinct, I think different dog breeds do have a right of existence, based on their specific history and characteristics. As Haraway (2008) has pointed out, the love of a breed that is prevalent in responsible breeders, also entails responsibilities from those breeders towards the breed. These responsibilities entail ensuring the health of the offspring rather than stressing certain aesthetic characteristics. I moreover agree with Derr (2004) that breeds unable to whelp naturally or to live normal lives outside an artificial environment should be modified or eliminated. This leads us to the difficult question too what extent are we allowed changing an animal to our taste even if it is not hurt or its quality of life is not diminished. Maybe we can use selective breeding to breed dogs that no longer need tails. In the article "Brave new birds: the use of 'animal integrity' in animal ethics" Bernice Bovenkerk et al. (2002) argue that the genetic alteration of animals hurts the animals as a species, even when the individual animal herself does not feel harm or has been engineered not to feel harm. This line of thought can be applied to extensive breeding as well: the notion of integrity is useful in an ethics of dog breeding, as it puts necessary restrictions on what we are allowed to do to them. I acknowledge, however, that this needs further elaboration and thought before it can be applied in this context.

\section{Conclusion}

The special location of dogs in the realm of natureculture, together with the unique characteristics of the bond between companion animal and man call for an application of a special set of ethical principles. First, mankind has a responsibility towards the dogs it houses: they should be treated with a high regard for their wellbeing. This means an extension of current laws, which in many countries still allow for several kinds of abuse, such as puppy mills. Next, specific responsibilities arise from the bond we have with specific animals, which exceed what is legally enforceable. We are obligated to provide for physical and mental needs of the dogs in our care, and since the relationship is one of trust, this trust should not be easily broken.

Acknowledgments I would like to thank Professor Ullrich Melle, Professor Kris Dierickx, and the anonymous commentators for their proofreading of this article and their valuable comments.

\section{References}

American Veterinary Medical Association (AVMA). (2002). US Pet ownership and demographics Sourcebook. Schaumburg, IL: American Veterinary Medical Association.

Bovenkerk, B., Brom, F. W. A., \& Bergh, B. J. (2002). Brave new birds. The use of animal integrity in animal ethics. Hastings Center Report, 32, 16-22.

Budiansky, S. (1997). The Covenant of the wild. London: Phoenix.

Burgess-Jackson, K. (1998). Doing right by our animal companions. The Journal of Ethics, 2, 159-185.

Callicott, J. B. (1998). 'Back together again' again. Environmental values, 7, 461-475.

Clark, S. R. L. (1995). Enlarging the community: Companion animals. In B. Almond (Ed.), Introducing applied ethics (pp. 318-330). Oxford, UK \& Cambridge, USA: Blackwell.

Clothier, S. (2002). Bones would rain from the sky: Deepening our relationships with dogs. New York: Warner Books.

Clutton-Brock, J. (1995). Origins of the dog: Domestication and early history. In J. Serpell (Ed.), The domestic dog: Its evolution, behaviour and interactions with people (pp. 7-20). Cambridge: Cambridge University Press. 
Clutton-Brock, J. (1999). A natural history of domesticated mammals. Cambridge: Cambridge University Press.

Coppinger, R., \& Coppinger, L. (2001). Dogs: A new understanding of Canine origin, behaviour and evolution. Chicago: The University of Chicago Press.

Derr, M. (2004). Dog's best friend: Annals of the dog-human relationship. Chicago: The University of Chicago Press.

Despret, V. (2004). The body we care for: Figures of anthropo-zoo-genesis. Body \& Society, 10(2-3), 111-134.

Endenburg, N. (2001). Animals as companions: Demographic, motivational and ethical aspects of companion animal ownership. Amsterdam: Thesis Publishers.

Francione, G. (2000). Introduction to animal rights: Your child or the dog?. Philadelphia: Temple University Press.

Gruen, L. (1993). Re-valuing nature. In E. R. Winkler \& J. R. Coombs (Eds.), Applied ethics. A reader (pp. 293-312). Oxford, UK \& Cambridge, USA: Blackwell.

Haraway, D. (1991). A cyborg manifesto: Science, technology, and socialist-feminism in the late twentieth century. In D. Haraway (Ed.), Simians, Cyborgs and women (pp. 149-181). London: Free Association Books.

Haraway, D. (2003). The companion species manifesto: Dogs, people and significant otherness. Chicago: Prickly Paradigm Press.

Haraway, D. (2008). When species meet. Minneapolis: University of Minnesota Press.

Hitt, J. (Ed.). (1991). The Harper's forum book: What are we talking about. New York: Citadel Press.

Johnson, L. E. (1991). A morally deep world: An essay on moral significance and environmental ethics. Cambridge: Cambridge University Press.

Koler-Matznick, J. (2002). The origin of the dog revisited. Anthrozoös, 15(2), 98-118.

Lorenz, K. (1988). Man meets dog. New York: Penguin Group.

Menache, S. (2001). Hunting and attachment to dogs in the Pre-Modern Period. In A. L. Podberscek, E. S. Paul, \& J. Serpell (Eds.), Companion animals \& us: Exploring the relationships between people \& pets (pp. 42-60). Cambridge: Cambridge University Press.

Midgley, M. (1984). Animals and why they matter. Athens: University of Georgia Press.

Podberscek, A. L., \& Gosling, S. (2001). Personality research on pets and their owners: Conceptual issues and review. In A. L. Podberscek, E. S. Paul, \& J. A. Serpell (Eds.), Companion animals \& us: Exploring the relationships between people \& pets (pp. 143-167). Cambridge: Cambridge University Press.

Rolston, H, I. I. I. (1993). Environmental ethics: Values in and duties to the natural world. In E. R. Winkler, R. Earl, J. R. Coombs, \& R. Jerrold (Eds.), Applied ethics. A Reader (pp. 271-292). Oxford, UK \& Cambridge, USA: Blackwell.

Serpell, J., Coppinger, R., \& Fine, A. H. (2003). The welfare of assistance and therapy animals: An ethical comment. In S. J. Armstrong \& R. G. Botzler (Eds.), The animal ethics reader (pp. 524-529). London and New York: Routledge.

Spencer, S., Decuypere, E., Aerts, S., \& De Tavernier, J. (2006). History and ethics of keeping pets: Comparison with farm animals. Journal of Agricultural and Environmental Ethics, 19, 17-25.

Stafford, K. (2007). The welfare of dogs. Dordrecht: Springer.

Tuan, Y. F. (1984). Dominance and affection: The making of pets. New Haven \& London: Yale University Press.

Vilà, C., Savolainen, P., Maldonado, J. E., Amorim, I. R., Rice, J. E., Honeycutt, R. L., et al. (1997). Multiple and ancient origins of the domestic dog. Science, 276(5319), 1687-1689. 